\title{
The Physiological Regulation of Skeletal Muscle Fatty Acid Supply and Oxidation During Moderate-Intensity Exercise
}

\author{
Gerrit van Hall ${ }^{1}$
}

Published online: 9 November 2015

(c) The Author(s) 2015. This article is published with open access at Springerlink.com

\begin{abstract}
Energy substrates that are important to the working muscle at moderate intensities are the non-esterified fatty acids (NEFAs) taken up from the circulation and NEFAs originating from lipolysis of the intramuscular triacylglycerol (IMTAG). Moreover, NEFA from lipolysis via lipoprotein lipase (LPL) in the muscle of the very-lowdensity lipoproteins and in the (semi) post-prandial state chylomicrons may also contribute. In this review, the NEFA fluxes and oxidation by skeletal muscle during prolonged moderate-intensity exercise are described in terms of the integration of physiological systems. Steps involved in the regulation of the active muscle NEFA uptake include (1) increased energy demand; (2) delivery of NEFA to the muscle; (3) transport of NEFA into the muscle by NEFA transporters; and (4) activation of the NEFAs and either oxidation or re-esterification into IMTAG. The increased metabolic demand of the exercising muscle is the main driving force for all physiological regulatory processes. It elicits functional hyperemia, increasing the recruitment of capillaries and muscle blood flow resulting in increased NEFA delivery and accessibility to NEFA transporters and LPL. It also releases epinephrine that augments adipose tissue NEFA release and thereby NEFA delivery to the active muscle. Moreover, NEFA transporters translocate to the plasma membrane, further increasing the NEFA uptake. The majority of the NEFAs taken up by the active muscle is oxidized and a minor
\end{abstract}

Gerrit van Hall

Gerrit.van.hall@regionh.dk

1 Clinical Metabolomics Core Facility, Department of Biomedical Sciences, Faculty of Health and Medical Sciences, Rigshospitalet, University of Copenhagen, Section 7652, 9 Blegdamsvej, 2100 Copenhagen, Denmark portion is re-esterified to IMTAG. Net IMTAG lipolysis occurs; however, the IMTAG contribution to total fat oxidation is rather limited compared to plasma-derived NEFA oxidation, suggesting a complex role and regulation of IMTAG utilization.

\section{Introduction}

Limitation of carbohydrate and lipid transfer from the microvascular system to the muscle cell occurs at the onset of exercise when the delivery and transport systems are not optimal and during continuous exercise above moderate intensities of $50 \%$ of maximal pulmonary oxygen uptake $\left(\mathrm{VO}_{2 \max }\right)$. At higher continuous workloads the extracellular substrate provision rate is not high enough and intracellular stored substrates must also be used, such as glycogen and intramuscular triacylglycerol (IMTAG) [1]. Due to complexity in the regulation of fat metabolism, it is still unknown what limits active muscle fat oxidation [2-4]. Various suggestions have been put forward, from limitation in the non-esterified fatty acids (NEFAs) delivery to the active muscle, to NEFA uptake into muscle or into the mitochondria or $\beta$-oxidation. In addition, the IMTAG not limited by delivery or uptake seems to be not utilized optimally as its pool is not even close to fully utilized [5].

The plasma NEFA concentration plays an important role in the NEFA uptake and subsequent oxidation by the active muscle. If the plasma NEFA concentration falls, the rate of muscle NEFA uptake and subsequent oxidation will decline as well. Conversely, an increase in the plasma NEFA concentration will increase the active muscle NEFA uptake and oxidation [6], similar to blood glucose and its uptake by the active muscle [7]. Adipose tissue release of NEFAs is the primary source of NEFAs by which the 
plasma NEFA concentration is sustained under post-absorptive conditions or increased during exercise [8]. Thus, the control of adipose tissue triacylglycerol (TAG) lipolysis and subsequent release of the NEFAs into the circulation has an important role in the regulation of muscle NEFA uptake and oxidation during exercise. The liver also plays an important role as it has a high NEFA uptake and, thereby, can affect the plasma NEFA concentration, albeit changes in liver NEFA uptake during exercise are not welldescribed [9]. Moreover, the NEFA delivery to the active muscles (NEFA concentration $\times$ plasma flow) is an even better determinant of the NEFA uptake than the NEFA concentration [10]. The blood flow to the active muscles increases several-fold upon exercise, primarily to increase oxygen supply, but it will also increase NEFA delivery. NEFA uptake from the plasma into the cytosol may occur to some extent via passive diffusion but over the past decades it has been shown that the majority of NEFA uptake occurs via facilitated transport and that muscle contraction induces plasma membrane fatty acid translocase (FAT/CD36) and fatty acid binding protein (FABPpm) translocation from the intracellular depots to the plasma membrane [11]. Therefore, NEFA transport into the muscle cells may be a potential regulatory and limiting step in NEFA utilization by muscle during exercise. Once in the cytosol, the NEFA is activated and can either be oxidized or stored in IMTAG. In this review, the control of human in vivo NEFA fluxes and subsequent oxidation by skeletal muscle during prolonged moderate-intensity exercise are described in terms of the integration of physiological systems (Fig. 1).

\section{Skeletal Muscle Non-Esterified Fatty Acid (NEFA) Oxidation During Exercise from Plasma-Derived NEFA and Intramuscular Triacylglycerol Lipolysis}

The regulation of the active muscle fatty acid uptake and subsequent oxidation is complex and may be differently regulated at low, moderate, and high exercise intensities and durations of exercise. Relatively few studies have directly determined human muscle NEFA handling during exercise, with most of the outstanding studies performed in the 1960-1970s [12-15], and even fewer studies have determined IMTAG involvement [6]. The limited, but remarkably consistent, available quantitative and kinetic information originates from prolonged moderate-intensity exercise. The regulation of the active muscle NEFA uptake can be defined by a four-step process, as depicted in Fig. 1, consisting of (1) an increased energy demand by the contracting muscle; (2) delivery of NEFA to the muscle; (3) transport of NEFA into the muscle by fatty acids transporters; and (4) activation of the fatty acids and either oxidation or re-esterified into intracellular lipids and stored into the IMTAG droplets located next to the mitochondria. A similar concept is generally acknowledged for glucose [7, 16] but differences seem to exist with respect to glycogen versus IMTAG as the intramuscular carbohydrate and fat energy stores, respectively.

The increased metabolic rate/demand of the active muscle is usually not considered to be a regulatory step. However, it is the main driving force for all physiological regulatory processes. It elicits functional hyperemia, increasing muscle blood flow, the number of perfused capillaries (recruitment), and hormone levels that affect adipose tissue NEFA release, and hence NEFA delivery to the active muscle. Moreover, resting skeletal muscle has very low energy expenditure and thus the demand for energy/adenosine triphosphate is small, implying that an increase in energy demand with exercise causes a surge for substrates that possibly creates a concentration gradient for NEFA between plasma, the interstitial space, cytosol, and entry in the mitochondria (Figs. 1, 2).

The resting muscle blood flow is low and $45-55 \%$ of all NEFA delivered to the muscle is taken up [10, 12, 15, 17] and is clearly dependent on the NEFA concentration [6]. This suggests that the facilitated muscle NEFA uptake is limited/saturable or that the lack of substrate demand and/ or re-esterification into intracellular lipids is low, reducing the NEFA gradient for uptake. During moderate-intensity exercise the blood flow to the active muscles increases linearly with the workload [18] and is easily 10- to 15 -fold higher during moderate-intensity exercise [17] than at rest, and with the increase in blood flow the blood transit time through the active muscle is substantially reduced $[19,20]$. The NEFA fractional extraction decreases to only $\sim 20 \%$ $[6,10,12-15,17]$ as compared to the $45-55 \%$ at rest, despite the massive increase in blood flow and reduced transit time. In addition, the NEFA factional extraction is the same over a wide range of plasma flows [12], i.e., exercise intensities, and is independent of the NEFA concentration over at least a threefold increase in NEFA concentration [6, 15]. Accordingly, the active muscle NEFA uptake is very closely and linearly related to the NEFA delivery, which is the NEFA concentration multiplied by the plasma flow to the active muscle [6, 10, 12-15, 17]. The same relationship is found between NEFA delivery and NEFA oxidation, since 85-100\% of the NEFA taken up by the active muscle is directly oxidized [6, 10, 12-14, 17]. Thus, during exercise the increase in NEFA uptake with delivery is dependent on the functional exercise hyperemia increase in blood flow. However, the linear increase of NEFA delivery and oxidation with the duration of prolonged moderate-intensity exercise is caused by the increase in NEFA concentration since the 


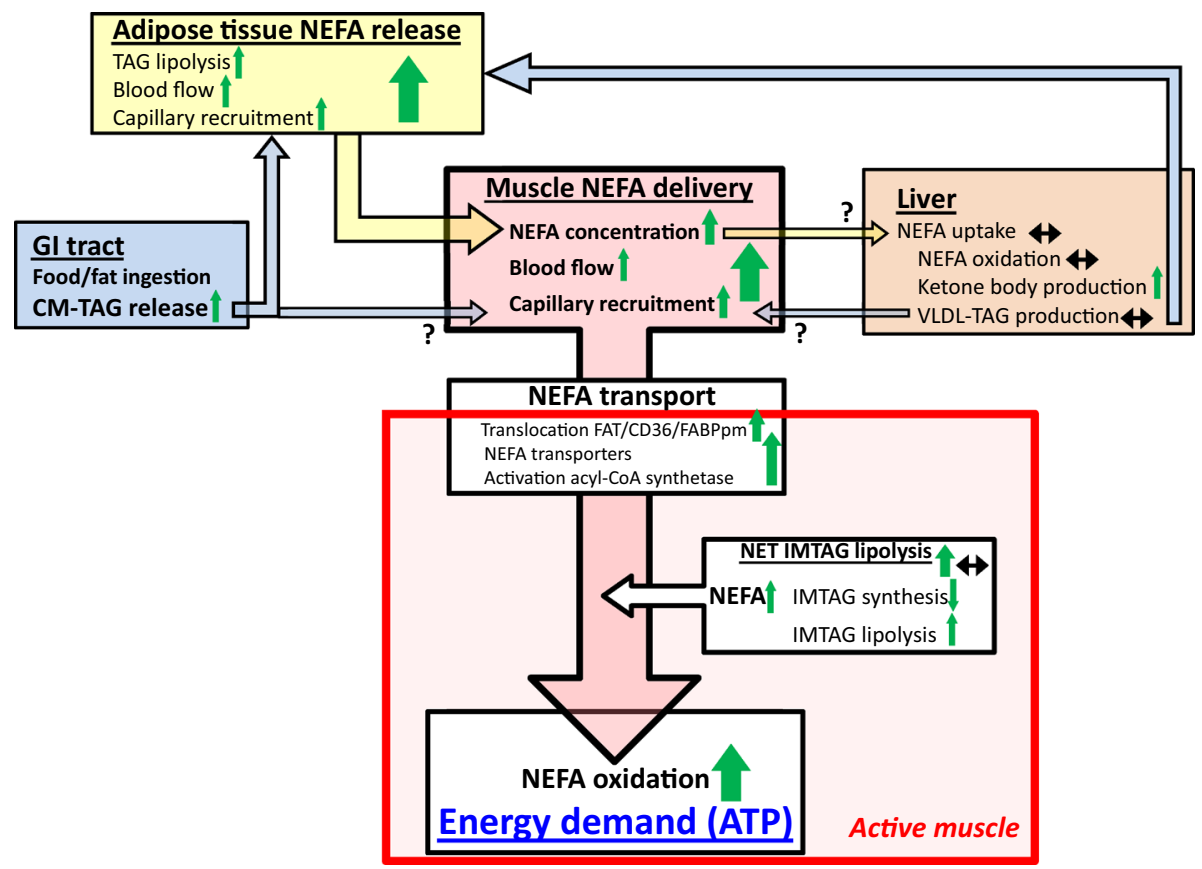

Fig. 1 Schematic representation of the control of non-esterified fatty acid (NEFA) fluxes and oxidation during exercise described in terms of the integration of physiological systems. Central in the scheme is the close linear relationship between the active muscle NEFA delivery and uptake/oxidation observed during continuous moderate-intensity exercise. The increase in NEFA delivery is caused by an increase in plasma flow that includes capillary recruitment, whereby a larger portion of the total available NEFA transport machinery becomes accessible. Plasma flow and capillary recruitment do not change much with continuous exercise at the same workload; hence, the increase in muscle NEFA delivery and uptake/oxidation with exercise duration is mainly mediated by the increase in NEFA concentration. The increase in the plasma NEFA concentration is facilitated by an increased release of NEFA from adipose tissue via increased adipose tissue triacylglycerol (TAG) lipolysis, adipose tissue blood flow, and capillary recruitment with epinephrine and possible atrial natriuretic peptide as the key regulators during exercise. Liver NEFA uptake at rest and during exercise is substantial but does not seem to change much with exercise, thus it does not have

plasma flow is largely unchanged during continuous exercise at the same intensity, emphasizing the important role of an increased adipose tissue NEFA release to enhance the plasma NEFA concentration driving the active muscle NEFA oxidation (Fig. 1 and Sect. 3) [8]. Consistent with these findings, an increase in the NEFA concentration via intralipid/heparin infusion increases the active muscle net NEFA uptake and fat oxidation [21], and even total fat oxidation at the relative high workload of $85 \%$ of $\mathrm{VO}_{2 \max }$ [22]. Conversely, if NEFA concentrations are lowered via nicotinic acid infusion, the plasma NEFA oxidation is reduced [23]. The capacity for muscle to achieve such a rapid and manyfold increase in NEFA uptake upon contraction is remarkable, particularly in view of the manyfold increase in NEFA delivery caused by the massive increase much effect on the plasma NEFA concentration. Mandatory, but likely not limiting for NEFA oxidation in healthy individuals, is the facilitated NEFA uptake by transporters and binding proteins of which fatty acid translocase (FAT/CD36) and fatty acid bounding protein $(\mathrm{FABPpm})$ translocate from the intracellular $\operatorname{depot}(\mathrm{s})$ to the plasma membrane with muscle contraction. NEFA from intramuscular TAG (IMTAG) is used during moderate-intensity exercise. The net degradation of IMTAG is caused by a decrease in IMTAG synthesis and maintained or increased lipolysis. Moreover, NEFA originating from either very-low-density lipoproteins (VLDL-TAG), or chylomicrons (CM-TAG) primarily in the fed state, may contribute to the active skeletal muscle NEFA oxidation. Dietary fat reaches the liver, adipose tissue, and skeletal muscle in the form of CM-TAG that undergoes lipolysis by lipoprotein lipase (LPL) and the resulting NEFAs are taken up and either oxidized or esterified by the active muscle (see also Fig. 2). The contribution of VLDL-TAG- and CMTAG-derived NEFA to the resting and active muscle energy requirements seems limited. ATP adenosine triphosphate, CoA coenzyme A, GI gastrointestinal

in blood flow and the substantially reduced time available for interaction with the NEFA transporters. Of course, the 10 - to 15 -fold increase in blood flow with moderate-intensity exercise is accompanied by an increased capillary recruitment $[19,20]$, also referred to as nutritive flow [24], increasing the accessibility to NEFA transporters and diffusion surface. In addition, contraction-induced translocation of NEFA transporters from the intracellular storage pool to the cell membrane $[11,25]$ plays an important role in enhancing muscle NEFA uptake capacity during exercise. Another contributing factor is likely the high energy demand of the active muscle creating a NEFA surge and increasing the gradient for NEFA movement from the mitochondrion to the cytosol and eventual plasma NEFA uptake. 


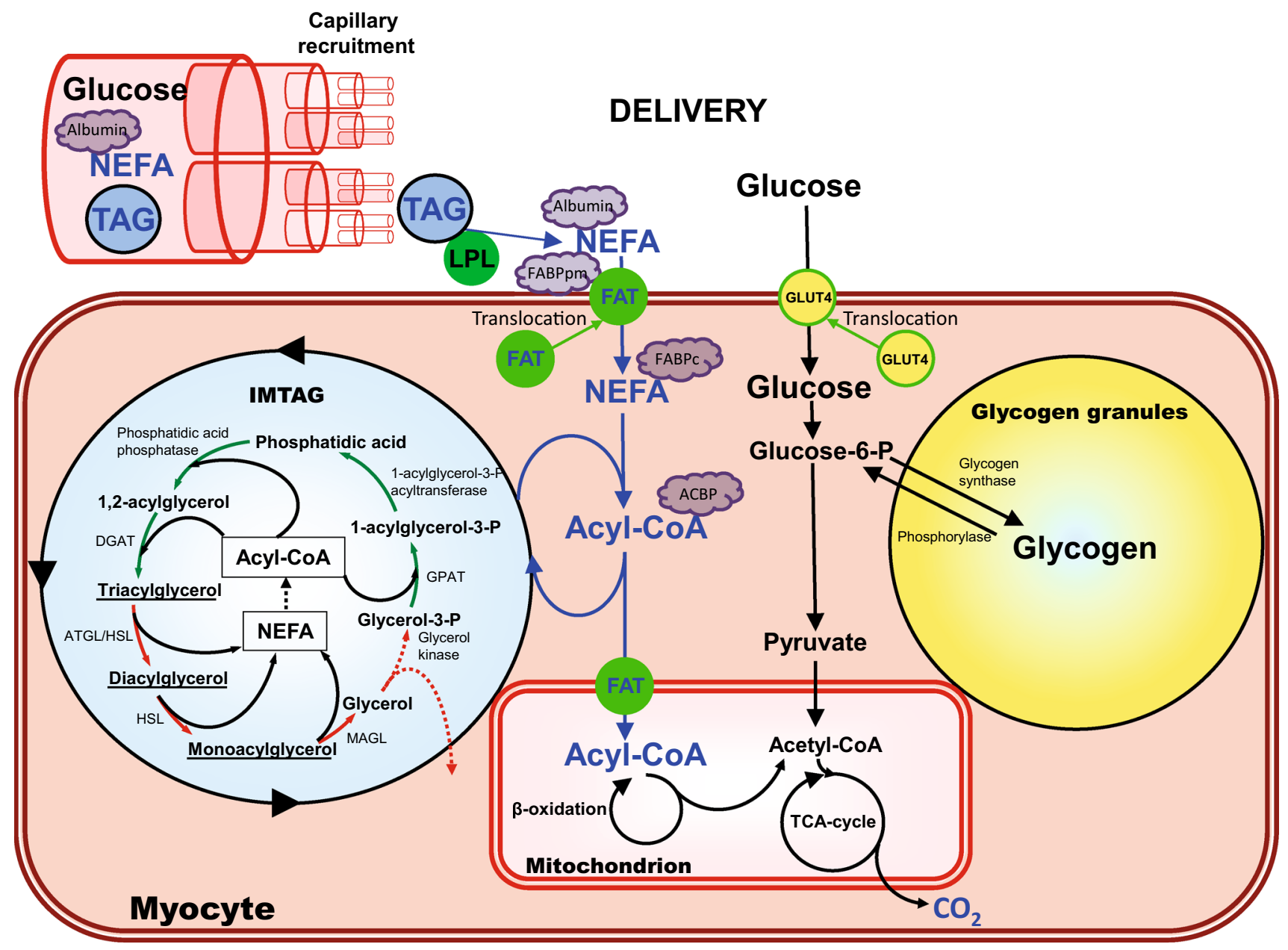

Fig. 2 Schematic representation of skeletal muscle energy metabolism. Two pathways in skeletal muscle energy oxidation during exercise can be recognized: the extracellular and intracellular substrate supply. The increase in the extracellular muscle energy supply during exercise is mediated via an increase in the blood substrate delivery of glucose from either carbohydrate intake or liver glycogenolysis and gluconeogenesis, non-esterified fatty acids (NEFA) mainly from adipose tissue, and chylomicron or very-lowdensity lipoproteins referred to as triacylglycerol (TAG) (see Fig. 1). The increase in delivery of these substrates to the active muscle is mediated by an increase in blood flow, including an increase in capillary recruitment, and substrate concentration. Transport of blood glucose into skeletal muscle is facilitated by glucose transporter-4 (GLUT4) and the long-chain NEFAs via fatty acid transporters (FAT), that also facilitates the transport of NEFA into the mitochondria. The fate of the glucose and NEFA taken up by skeletal muscle is oxidation or storage into glycogen or TAG, respectively. The

Over recent decades there has been considerable debate regarding whether NEFA transport across the plasma membrane occurs via passive diffusion or via facilitated transport by means of membrane-associated proteins. The physical properties of NEFA with a non-polar carbon chain and the polar head group would make passive diffusion possible, and this is seemingly supported by the abovedescribed connection between NEFA concentration and intracellular energy supply during exercise is immediately increased, mainly via a fast breakdown of glycogen crucial to cover the instantaneously manyfold increase in energy demand going from rest to exercise. The rate of glycogen breakdown decreases with exercise duration, and glucose uptake and subsequent oxidation and later fatty acid oxidation increases. The increase in NEFA availability from intramuscular triacylglycerol (IMTAG) breakdown during exercise is mediated by a reduction in NEFA re-esterification and possibly an increase in IMTAG lipolysis. The role and regulation of the muscle IMTAG turnover rate is unknown. $A C B P$ acyl-CoA binding protein, $A T G L$ adipose tissue triglyceride lipase, CoA coenzyme A, DGAT diacylglycerol acyltransferase, $F A B P$ fatty acid binding protein ( $p m$ plasma membrane, $c$ cytosolic), $F A B P m$ fatty acid binding protein, GPAT glycerol-3-phosphate acyltransferase, $H S L$ hormone sensitive lipase, $L P L$ lipoprotein lipase, $M A G L$ monoacylglycerol lipase, $T C A$ tricarboxylic acid

active muscle NEFA uptake. However, over the past decade it has been shown that the majority of NEFA is transported across the cell membrane via protein-mediated mechanisms. Moreover, some of these fatty acid transporters demonstrate reversible translocation, from the intracellular storage pool to the cell membrane $[11,25]$ under the influence of contraction and insulin, analogous to the well-described glucose transporter type-4 (GLUT-4) 
translocation for muscle glucose uptake [7, 16]. Furthermore, studies with FAT/CD36 knockout or overexpressing mice $[26,27]$ have lent support to the conclusion that these fatty acid transporters critically regulate skeletal muscle fuel selection, performance, and training-induced adaptation of NEFA oxidation [26, 27]. However, while these studies undoubtedly demonstrate the importance of NEFA transporters in skeletal muscle NEFA transport from the plasma into the active muscle, they do not provide information on whether these transporters are rate limiting for NEFA oxidation. The well-described human in vivo increase in NEFA oxidation with exercise duration and the close linear relationship between NEFA delivery and uptake into the active muscle suggests that NEFA uptake is not solely controlled by the rate of sarcolemmal transport. This does not exclude an important regulatory role for fatty acid transporters and translocation of resting muscle NEFA uptake and its role in NEFA clearance from the circulation, particularly after food ingestion and during exercise in untrained individuals or patients with, for example, type 2 diabetes mellitus.

Once NEFA is taken up by the active muscle cell it can be oxidized or incorporated into intracellular lipid-like membranes and/or re-esterified and stored into IMTAG. The rate of resting muscle NEFA re-esterification into IMTAG is high $[6,28,29]$, accounting for $\sim 50$ to $60 \%$ of the total NEFA uptake [6, 30]. During exercise the majority $(85-100 \%)$ of NEFA taken up is directly oxidized $[6,10,12-14,17]$ and the rate of re-esterification into IMTAG is reduced, accounting for $\sim 10 \%$ of the total NEFA uptake [6]. A net IMTAG utilization with prolonged moderate-intensity exercise is observed for mixed muscle as a result of a reduced rate of IMTAG synthesis [6] and potentially an increased IMTAG lipolysis by adipose tissue TAG lipase responsible for the contraction-induced IMTAG lipolysis [31, 32]. However, in contrast to glycogen, the mixed muscle IMTAG store does not seem to be utilized to a large extent (20-50\% of the pre-exercise total IMTAG [5, 6, 33-35]), and this was even the case after $12 \mathrm{~h}$ of exercise [36]. The mixed muscle net IMTAG breakdown during exercise may underestimate IMTAG utilization by the different fiber types since only type I fibers have been suggested to utilize IMTAG during $2 \mathrm{~h}$ of 60 and $75 \%$ of $\mathrm{VO}_{2 \max }$ bicycling exercise [37, 38] as well as after $2 \mathrm{~h}$ of highintensity knee-extensor exercise [39]. Moreover, type II fibers have a much lower IMTAG content, but this does not decrease during exercise [37-39]. The high IMTAG synthesis rate that is reduced but still continues during muscle contraction together with a seemingly sub-maximal utilization rate suggests that the role of IMTAG is not solely as a rapidly responsive intracellular energy store such as glycogen, particularly since the higher circulatory
NEFA oxidation shows that the NEFA oxidation capacity of mitochondria is not limiting for IMTAG utilization.

\section{Skeletal Muscle NEFA Supply from Adipose Tissue Fat Lipolysis During Exercise}

Prolonged moderate-intensity exercise in the post-absorptive state causes a continuous increase in the NEFA concentration and oxidation with exercise duration [10, 17, 34, 40]. Moreover, the continuous increase in the NEFA concentration with duration of exercise implies that the rate of NEFA entering the circulation is higher than the uptake rate of the active muscles. Most of the circulatory NEFAs originate from TAG lipolysis in adipose tissue. Direct measurements across abdominal subcutaneous adipose tissue show a substantial increase in NEFA release at low exercise intensities [41]. Moreover, no or only a modest increase in adipose tissue NEFA release is found with higher exercise intensity $[9,41]$. Therefore, low-intensity exercise provides an adequate stimulus for abdominal adipose tissue NEFA mobilization. The release of NEFA from subcutaneous adipose tissues depends on the relative contribution of three processes: (1) adipose tissue TAG lipolysis; (2) the rate of adipose tissue NEFA re-esterification into TAG; and (3) adipose tissue blood flow. The adipose tissue NEFA uptake is undetectable at rest [9, 42] and during exercise [9]; hence, NEFA re-esterification does not play an important role and the increase in adipose tissue NEFA release can be attributed to an increase in TAG lipolysis. However, the exercise-induced subcutaneous adipose tissue blood flow [9, 41, 43] and likely capillary recruitment $[44,45]$ plays a crucial role in the increase in NEFA release during exercise. The difference between the arterial and adipose tissue venous NEFA concentration is very similar at rest and during exercise. Therefore, the 2- to 3 -fold increase in adipose tissue blood flow during exercise is responsible for the increase in the circulatory rate of NEFA [9]. The importance of adipose tissue blood flow for NEFA release is substantiated by observations in patients with type 2 diabetes who exhibit an exercise-stimulated adipose tissue lipolysis but in whom a high fraction of the liberated NEFA caused by TAG lipolysis could not be released into the circulation since the exercise-induced increase in adipose tissue blood flow was much smaller than in healthy subjects [46]. The exercise-induced increase in adipose tissue lipolysis and blood flow has mainly been attributed to the elevated catecholamine concentration with exercise [47, 48]. Moreover, circulating epinephrine is more important than sympathetic nervous activity for the stimulation of adipose tissue lipolysis during exercise $[49,50]$, albeit differences in various adipose tissue depots may exist [51]. Whereas epinephrine may be 
primarily responsible for the exercise-induced increase in lipolysis, selective $\beta$-adrenergic blockage does not completely prevent the increase in lipolysis [47, 48, 52], suggesting that other mediators play a role in the stimulation of lipolysis during exercise. Atrial natriuretic peptide is a potential candidate since there is evidence that it stimulates adipose tissue lipolysis and is produced during exercise in an intensity-dependent manner [53-55]. Growth hormone and cortisol increase adipose tissue lipolysis [56-58] and are potentially involved in the regulation of adipose tissue lipolysis during exercise. Growth hormone usually increases with exercise but its effect on lipolysis is not manifested until after 1-2 $\mathrm{h}$ of exercise [59] and thus is likely to become more important during prolonged exercise or be involved in the enhanced lipolysis during recovery from exercise [59]. Interestingly, 4 weeks of high-dose growth hormone supplementation raised the basal lipolytic rate substantially but did not change the exercise-induced increase in lipolysis during $30 \mathrm{~min}$ of exercise at $65 \%$ of $\mathrm{VO}_{2 \max }$ [60], suggesting that growth hormone does not play an important role in the exercise-induced increase in adipose tissue lipolysis. Cortisol may increase to some extent with prolonged exercise [61] but this does not coincide with the rapid increase of adipose tissue TAG lipolysis upon the start of exercise. It has been suggested that infused cortisol and growth hormone stimulate systemic and adipose tissue lipolysis in an additive manner; however, it has to be mentioned that the cortisol levels were substantially higher than seen for any form of exercise [62]. Interleukin-6 (IL-6) is produced by skeletal muscle during exercise in increasing amounts with increased duration [63], and when recombinant human IL-6 (rhIL-6) is infused it increases systemic lipolysis [64]. Thus, IL-6 could be a signal from muscle to adipose tissue to coordinate the NEFA supply from adipose tissue with demand from the exercising muscle. However, concomitant IL-6 infusion and exercise fails to raise fatty acid delivery or oxidation [65], and at rest rhIL-6 infusion does not increase adipose NEFA release but does increase NEFA release from skeletal muscle [66]. Therefore, IL-6 is not a regulator for the increased adipose tissue NEFA release during exercise, but may be involved in skeletal muscle TAG utilization during exercise.

It should be noted that most research is performed in the post-absorptive state and little information exists on adipose tissue NEFA release during exercise in the postprandial state. Ingestion of a mixed meal $1 \mathrm{~h}$ before exercise causes much lower plasma NEFA concentrations but does not influence adipose tissue lipolysis [67]. However, the adipose tissue net NEFA release is substantially lower during exercise in the post-prandial than in the fasted state as a result of the high adipose tissue NEFA uptake and reesterification into TAG. In addition, the exercise-induced increase in adipose tissue blood flow is absent post-prandially [67]. Thus, the decrease in the muscle fat oxidation during exercise after a mixed meal may in part be caused by reduced NEFA delivery to the active muscle as a consequence of the decreased fatty acid mobilization from adipose tissue.

\section{Skeletal Muscle Fatty Acid Supply from Non- Adipose Tissue Fat Lipolysis During Exercise}

Lipolysis of circulating TAG-rich lipoproteins in the capillaries of skeletal muscle is a source of NEFA for uptake and either oxidation or re-esterification in the active muscles. The majority of TAG-rich lipoproteins are the verylow-density lipoproteins (VLDL-TAG) produced in the liver in the fed and fasted state and contains one molecule of apolipoprotein $\mathrm{B}_{100}$ and 5-25,000 TAG molecules in its core [68]. In the fed state chylomicron TAG (CM-TAG) is secreted by the intestines and begins to appear in the blood approximately $1 \mathrm{~h}$ after the oral fat intake. The large CMTAG contain apolipoprotein $\mathrm{B}_{48}$ and are very rich in TAG with a half-life estimated at 5 min [69]. Both VLDL-TAG and CM-TAG undergo lipolysis by lipoprotein lipase (LPL) anchored to heparin sulphate on the endothelial surfaces in muscle. The affinity of LPL for CM-TAG is reported to be 50-fold higher than for VLDL-TAG; however, this is balanced by the usually much higher concentration of VLDL-TAG. Moreover, complex differences exist between tissues in LPL expression, activity, and specific nutritional regulation [70, 71]. Circulating CMTAGs are suggested to provide a more readily available source of NEFA for muscle oxidation than VLDL-TAG [72]. However, this obviously requires oral fat intake before or during the exercise, something that is not common practice among athletes. A key problem in the quantification of muscle CM-TAG and VLDL-TAG lipolysis is the technical difficulty of measuring TAG extraction across a muscle, a problem that is exaggerated during exercise when blood flow to the active muscle increases manyfold [73]. In general, no significant extraction of TAG, CMTAG, and VLDL-TAG by the active or inactive muscle can be determined in the fasted state $[10,73,74]$. However, in spite of this inability to measure small quantities of muscle TAG extraction, the potential amount of NEFA for the muscle can contribute substantially to the total fat oxidation. Enevoldsen et al. [75] did not observe leg TAG uptake $1 \mathrm{~h}$ after meal ingestion, but during the following hour a significant TAG uptake of $\sim 45 \mu \mathrm{mol} / \mathrm{min}$ was observed in only the exercising leg during 1 hour of cycle ergometer exercise at $50 \%$ of $\mathrm{VO}_{2 \max }$. The NEFA uptake in the exercising leg was of similar magnitude; in other words, the NEFA uptake was only one-third of the TAG uptake in 
NEFA equivalents. Moreover, these results suggest that exercise per se increases muscle LPL activity, which has previously been shown not to occur via more direct muscle LPL activity measurements [76, 77]. However, Enevoldsen et al. [75] argued that not all capillaries are recruited in the resting muscle, but that the functional hyperemia of the active muscle increases the number of perfused capillaries $[24,78]$ and, hence, the accessible amount of LPL as depicted in Fig. 2. Evidence against a considerable contribution of circulating TAG to active muscle fat oxidation include findings that in the fasted state the muscle NEFA uptake and oxidation account for most of the fat oxidation $[6,10,12,14,15,17]$ and also for the majority of wholebody fat oxidation [37, 79], which suggests that relatively little of the oxidation of NEFAs originates from CM-TAG and VLDL-TAG. More research is needed to quantify the contribution of VLDL-TAG and CM-TAG to muscle fat oxidation and whether nutritional interventions before or during exercise can enhance its utilization without compromising performance.

\section{Discussion}

In 1991, Weibel et al. [80] proposed the concept of symmorphosis. The concept states that animals and humans should be designed economically, i.e., that structural design should be matched to functional demand, or, in other words, no more structure is built and maintained than is required to meet functional demand. During very highintensity exercise the concept of symmorphosis is put to the test as it is ultimately the active muscle that sets the aerobic demand because more than $90 \%$ of energy is spent in the active muscle. The limitation of carbohydrate and lipid transfer from the microvascular system to the muscle cell seems to be reached at the onset of exercise and during continuous exercise above moderate intensities $>50 \%$ of $\mathrm{VO}_{2 \text { max }}$. At higher workloads, intracellular stored substrates must be used for oxidation [1]. Glycogen can supply pyruvate up to the limit of maximal oxidation rates by the mitochondria, plus a further several-fold increase to fuel 'anaerobic' glycolysis. The question is, does IMTAG have a similar role to glycogen? The IMTAG droplets and the glycogen granules contain roughly equal amounts of energy and are in close vicinity to the mitochondria (Fig. 2). Indeed, IMTAG is utilized during moderate-intensity exercise, but in substantially lower quantities than plasma NEFA oxidation. In addition, when NEFA concentration/availability is low the IMTAG utilization is increased during moderate-intensity exercise, but it cannot compensate for the reduction in plasma NEFA oxidation $[23,81]$. In other words, total fat oxidation by the active muscle is reduced. Enhanced muscle glycogen utilization has to cover the reduced NEFA oxidation when NEFA availability is low; if not, the ability to perform prolonged exercise is impaired [82]. It also demonstrates that NEFA uptake into the mitochondria and oxidation does not limit IMTAG utilization. Also different from glycogen is that IMTAG has an energy-consuming turnover rate of about $1-1.5$ of the total IMTAG pool size/day, and, although IMTAG synthesis during exercise is reduced, it remains at a substantial rate [6] and it seems that the rate of complete IMTAG degradation to NEFA cannot be up-regulated very quickly in response to changes in demand. Therefore, it appears that IMTAG does not have the role of a rapidly responsive, directly available fat energy source without availability or transport limitations during exercise according to the concept of symmorphosis. Our knowledge of IMTAG turnover, lipolytic rate, and net utilization is almost completely lacking under conditions such as food intake and early versus late and moderate- versus highintensity exercise, which makes any hypothesis on the role of IMTAG daunting. Perhaps IMTAG with its continuous high turnover rate may act as a 'buffer' for intramyocellular NEFAs, which in a high(er) concentration may be toxic $[83,84]$ and in a low(er) concentration compromises energy provision to the active muscles. The high synthesis and degradation rates for IMTAG mean it is able to fulfil this dual task. This role could be more important in slow twitch fibers with higher mitochondrial and IMTAG content and NEFA oxidation during exercise [37-39]. Research on fiber type differences and muscle NEFA content, IMTAG turnover, and NEFA transporters under various exercise conditions are needed to understand the role of IMTAG, particularly in various metabolic diseases.

Studies on patients with myophosphorylase deficiency or McArdle disease (also known as glycogen storage disease $\mathrm{V}$ ) provide important insights into the role of circulatory glucose and NEFA versus intramuscular stored glycogen and IMTAG. Patients with McArdle disease cannot break down muscle glycogen and are characterized by a severely impaired exercise capacity, particularly during the initial $10 \mathrm{~min}$ of exercise, which is followed by sudden improvement in the exercise capacity referred to as the 'second wind' phenomenon. This second wind is attributed to an increase in blood glucose uptake [85] that is higher than in healthy individuals and is mediated by an increase in the number of GLUT-4 proteins or the localization in the membrane of GLUT-4 [86] and enhanced muscle blood flow [87]. Moreover, glucose supplementation greatly improves performance after $\sim 5$ min of exercise [88]. These findings underscore the important role of muscle glycogen to cover much of the instant manyfold increase in energy demand and the initial minute when the blood flow to the muscle is not yet fully increased to supply the necessary oxygen for substrate oxidation [18]. 
Moreover, the rate of glucose uptake by the active muscles is far lower than the glucose/glycogen substrate demand for high exercise intensities. During exercise, patients with McArdle disease oxidize more circulatory NEFA than healthy controls exercising at the same intensity [89], but enhanced availability via intralipid infusion does not improve performance [90]. The low rate of acetyl-coenzyme A (CoA) production from glycolysis cannot be replaced by a high rate of acetyl-CoA production from the $\beta$-oxidation likely related to reduced availability of oxaloacetate, tricarboxylic acid cycle intermediates, since ample pyruvate and/or amino acids are mandatory. Thus, glycogen is required to oxidize NEFA, whether from circulatory NEFA or IMTAG, with quantities the same as low to moderate intensities in healthy individuals required for higher exercise intensities in patients with McArdle disease.

Our understanding of fat metabolism during exercise is incomplete, not least the role of IMTAG. Apart from endurance training, most attempts to increase fat/NEFA oxidation to improve performance with the rationale of sparing the carbohydrates for high-intensity exercise have been rather unsuccessful. Endurance training increases the capacity of the muscle to take up NEFA [91], potentially by enhancing the number of capillaries and/or recruitment and the muscle NEFA oxidative capacity [91]. But how can we elevate the plasma NEFA concentration, which is an important determinant of the active muscle NEFA uptake and subsequent oxidation? Might there be an easier or additive way to enhance fat oxidation during exercise? This may be possible via ingestion of small quantities of fat before and/or during prolonged moderate-intensity exercise, which may aid the endogenous increase in NEFA release from adipose tissue, particularly during the initial 30 min of exercise when the NEFA concentration slightly decreases. However, oral glucose intake has been proven to enhance performance/endurance and should also be provided, but it will reduce the adipose tissue rate of lipolysis. Information is lacking but the simultaneous ingestion of starch with relatively small quantities of fat may potentially achieve the best results for both carbohydrate and fat utilization and, hence, performance.

\footnotetext{
Acknowledgments This article was published in a supplement supported by the Gatorade Sports Science Institute (GSSI). The supplement was guest edited by Lawrence L. Spriet who attended a meeting of the GSSI expert panel (XP) in March 2014 and received honoraria from GSSI for his participation in the meeting. He received no honoraria for guest editing the supplement. Dr Spriet selected peer reviewers for each paper and managed the process. Gerrit van Hall also attended the meeting of the GSSI XP in March 2014 and received honoraria from the GSSI, a division of PepsiCo, Inc., for his meeting participation and the writing of this manuscript. The views expressed in this manuscript are those of the author and do not necessarily reflect the position or policy of PepsiCo, Inc. The author's work was
}

supported by The Copenhagen Muscle Research Centre which was funded by a grant from the Danish National Research Foundation (Grant No. 504-14), the Danish National Research Foundation (Grant No. 02-512-55), Novo Nordisk Foundation, and the Danish Medical Research Council.

Open Access This article is distributed under the terms of the Creative Commons Attribution 4.0 International License (http:// creativecommons.org/licenses/by/4.0/), which permits unrestricted use, distribution, and reproduction in any medium, provided you give appropriate credit to the original author(s) and the source, provide a link to the Creative Commons license, and indicate if changes were made.

\section{References}

1. Hoppeler H, Weibel ER. Limits for oxygen and substrate transport in mammals. J Exp Biol. 1998;201:1051-64.

2. Sahlin K, Harris RC. Control of lipid oxidation during exercise: role of energy state and mitochondrial factors. Acta Physiol. 2008;194:283-91.

3. Jeppesen J, Kiens B. Regulation and limitations to fatty acid oxidation during exercise. J Physiol. 2012;590:1059-68.

4. Spriet L. New Insights into the interaction of carbohydrate and fat metabolism during exercise. Sports Med. 2014;2(44):87-96.

5. Zehnder M, Christ E, Ith M, et al. Intramyocellular lipid stores increase markedly in athletes after 1.5 days lipid supplementation and are utilized during exercise in proportion to their content. Eur J Appl Physiol. 2006;98:341-54.

6. Sacchetti M, Saltin B, Osada T, et al. Intramuscular fatty acid metabolism in contracting and non-contracting human skeletal muscle. J Physiol. 2002;540:387-95.

7. Jensen TE, Richter EA. Regulation of glucose and glycogen metabolism during and after exercise. J Physiol. 2012;590: 1069-76.

8. Frayn KN. Fat as a fuel: emerging understanding of the adipose tissue-skeletal muscle axis. Acta Physiol. 2010;199:509-18.

9. van Hall G, Bülow J, Sacchetti M, et al. Regional fat metabolism in human splanchnic and adipose tissues; the effect of exercise. J Physiol. 2002;543:1033-46.

10. Turcotte L, Richter E, Kiens B. Increased plasma FFA uptake and oxidation during prolonged exercise in trained vs. untrained humans. Am J Physiol. 1992;262:E791-9.

11. Schwenk RW, Holloway GP, Luiken JJFP, et al. Fatty acid transport across the cell membrane: Regulation by fatty acid transporters. Prostagland Leukot Essent Fatty Acids. 2010;82:149-54.

12. Havel RJ, Pernow B, Jones NL. Uptake and release of free fatty acids and other metabolites in the legs of exercising men. J Appl Physiol. 1967;23:90-9.

13. Hagenfeldt L, Wahren J. Human forearm muscle metabolism during exercise. II. Uptake, release and oxidation of individual FFA and glycerol. Scand J Clin Lab Invest. 1968;21:263-76.

14. Hagenfeldt L, Wahren J. Human forearm muscle metabolism during exercise. VII. FFA uptake and oxidation at different work intensities. Scand J Clin Lab Invest. 1972;30:429-36.

15. Ahlborg G, Felig P, Hagenfeldt L, et al. Substrate turnover during prolonged exercise in man: splanchnic and leg metabolism of glucose, free fatty acids, and amino acids. J Clin Invest. 1974;53:1080-90.

16. Wasserman DH, Kang L, Ayala JE, et al. The physiological regulation of glucose flux into muscle in vivo. J Exp Biol. 2011;214:254-62. 
17. van Hall G, Sacchetti M, Rådegran G, et al. Human skeletal muscle fatty acid and glycerol metabolism during rest, exercise and recovery. J Physiol. 2002;543:1047-58.

18. Rådegran G, Saltin B. Muscle blood f low at onset of dynamic exercise in humans. Am J Physiol. 1998;274:H314-22.

19. Saltin B. Hemodynamic adaptations to exercise. Am J Cardiol. 1985;55:42D-7D.

20. Calbet JAL, Holmberg H-C, Rosdahl H, et al. Why do arms extract less oxygen than legs during exercise? Am J Physiol. 2005;289:R1448-58.

21. Odland LM, Heigenhauser GJF, Wong D, et al. Effects of increased fat availability on fat-carbohydrate interaction during prolonged exercise in men. Am J Physiol. 1998;274:R894-902.

22. Romijn JA, Coyle EF, Sidossis LS, et al. Relationship between fatty acid delivery and fatty acid oxidation during strenuous exercise. J Appl Physiol. 1995;79:1939-45.

23. van Loon LJC, Thomason-Hughes M, Constantin-Teodosiu D, et al. Inhibition of adipose tissue lipolysis increases intramuscular lipid and glycogen use in vivo in humans. Am J Physiol. 2005;289:E482-93.

24. Clark M, Rattigan S, Barrett E. Nutritive blood flow as an essential element supporting muscle anabolism. Curr Opin Clin Nutr Metab Care. 2006;9:185-9.

25. Jain SS, Chabowski A, Snook LA, et al. Additive effects of insulin and muscle contraction on fatty acid transport and fatty acid transporters, FAT/CD36, FABPpm, FATP1, 4 and 6. FEBS Lett. 2009;583:2294-300.

26. Holloway GP, Jain SS, Bezaire V, et al. FAT/CD36-null mice reveal that mitochondrial $\mathrm{FAT} / \mathrm{CD} 36$ is required to upregulate mitochondrial fatty acid oxidation in contracting muscle. Am J Physiol. 2009;297:R960-7.

27. McFarlan JT, Yoshida Y, Jain SS, et al. In vivo, fatty acid translocase (CD36) critically regulates skeletal muscle fuel selection, exercise performance, and training-induced adaptation of fatty acid oxidation. J Biol Chem. 2012;287:23502-16.

28. Kanaley JA, Shadid S, Sheehan MT, et al. Relationship between plasma free fatty acid, intramyocellular triglycerides and longchain acylcarnitines in resting humans. J Physiol. 2009;587: 5939-50.

29. Bergman BC, Perreault L, Hunerdosse DM, et al. Increased intramuscular lipid synthesis and low saturation relate to insulin sensitivity in endurance-trained athletes. J Appl Physiol. 2010; 108:1134-41.

30. Bucci M, Borra R, Någren K, et al. Human obesity is characterized by defective fat storage and enhanced muscle fatty acid oxidation, and trimetazidine gradually counteracts these abnormalities. Am J Physiol. 2011;301:E105-12.

31. Alsted TJ, Ploug T, Prats C, et al. Contraction-induced lipolysis is not impaired by inhibition of hormone-sensitive lipase in skeletal muscle. J Physiol. 2013;591:5141-55.

32. Huijsman E, van de Par C, Economou C, et al. Adipose triacylglycerol lipase deletion alters whole body energy metabolism and impairs exercise performance in mice. Am J Physiol. 2009; 297:E505-13.

33. Hurley BF, Nemeth PM, Martin WH, et al. Muscle triglyceride utilization during exercise: effect of training. J Appl Physiol. 1986;60:562-7.

34. Watt MJ, Heigenhauser GJF, Dyck DJ, et al. Intramuscular triacylglycerol, glycogen and acetyl group metabolism during $4 \mathrm{~h}$ of moderate exercise in man. J Physiol. 2002;541:969-78.

35. Larson-Meyer DE, Newcomer BR, Hunter GR. Influence of endurance running and recovery diet on intramyocellular lipid content in women: a 1H NMR study. Am J Physiol. 2002;282: E95-106.

36. Essén B. Intramuscular substrate utilization during prolonged exercise. Ann N Y Acad Sci. 1977;301:30-44.
37. van Loon LJC, Koopman R, Stegen JHCH, et al. Intramyocellular lipids form an important substrate source during moderate intensity exercise in endurance-trained males in a fasted state. J Physiol. 2003;553:611-25.

38. De Bock K, Richter EA, Russell AP, et al. Exercise in the fasted state facilitates fibre type-specific intramyocellular lipid breakdown and stimulates glycogen resynthesis in humans. J Physiol. 2005;564:649-60.

39. Prats C, Gomez-Cabello A, Nordby P, et al. An optimized histochemical method to assess skeletal muscle glycogen and lipid stores reveals two metabolically distinct populations of type I muscle fibers. PLoS One. 2013;8:e77774.

40. Coyle E, Jeukendrup A, Wagenmakers A, et al. Fatty acid oxidation is directly regulated by carbohydrate metabolism during exercise. Am J Physiol. 1997;273:E268-75.

41. Al Mulla N, Simonsen L, Bülow J. Post-exercise adipose tissue and skeletal muscle lipid metabolism in humans: the effects of exercise intensity. J Physiol. 2000;524:919-28.

42. Coppack SW, Persson M, Judd RL, et al. Glycerol and nonesterified fatty acid metabolism in human muscle and adipose tissue in vivo. Am J Physiol. 1999;276:E233-40.

43. Thompson D, Karpe F, Lafontan M, et al. Physical activity and exercise in the regulation of human adipose tissue physiology. Physiol Rev. 2012;92:157-91.

44. Tobin L, Simonsen L, Bülow J. Real-time contrast-enhanced ultrasound determination of microvascular blood volume in abdominal subcutaneous adipose tissue in man. Evidence for adipose tissue capillary recruitment. Clin Physiol Funct Imaging. 2010;30:447-52.

45. Sjøberg KA, Rattigan S, Hiscock N, et al. A new method to study changes in microvascular blood volume in muscle and adipose tissue: real-time imaging in humans and rat. Am J Physiol. 2011;301:H450-8.

46. Simonsen L, Henriksen O, Enevoldsen L, et al. The effect of exercise on regional adipose tissue and splanchnic lipid metabolism in overweight type 2 diabetic subjects. Diabetologia. 2004;47:652-9.

47. Arner P, Kriegholm E, Engfeldt P, et al. Adrenergic regulation of lipolysis in situ at rest and during exercise. J Clin Invest. 1990;85:893-8.

48. Samra J, Simpson E, Clark M, et al. Effects of epinephrine infusion on adipose tissue: interactions between blood flow and lipid metabolism. Am J Physiol. 1996;271:E834-9.

49. Stallknecht B, Lorentsen J, Enevoldsen LH, et al. Role of the sympathoadrenergic system in adipose tissue metabolism during exercise in humans. J Physiol. 2001;536:283-94.

50. de Glisezinski I, Larrouy D, Bajzova M, et al. Adrenaline but not noradrenaline is a determinant of exercise-induced lipid mobilization in human subcutaneous adipose tissue. J Physiol. 2009;587:3393-404.

51. Manolopoulos KN, Karpe F, Frayn KN. Marked resistance of femoral adipose tissue blood flow and lipolysis to adrenaline in vivo. Diabetologia. 2012;55:3029-37.

52. Moro C, Crampes F, Sengenes C, et al. Atrial natriuretic peptide contributes to the physiological control of lipid mobilization in humans. FASEB J. 2004;18:908-10.

53. Birkenfeld AL, Boschmann M, Moro C, et al. Lipid Mobilization with physiological atrial natriuretic peptide concentrations in humans. J Clin Endocrinol Metab. 2005;90:3622-8.

54. Lafontan M, Moro C, Sengenes C, et al. An unsuspected metabolic role for atrial natriuretic peptides: the control of lipolysis, lipid mobilization, and systemic nonesterified fatty acids levels in humans. Arterioscler Thromb Vasc Biol. 2005;25:2032-42.

55. Moro C, Pillard F, de Glisezinski I, et al. Sex differences in lipolysis-regulating mechanisms in overweight subjects: effect of exercise intensity. Obesity. 2007;15:2245-55. 
56. Samra JS, Clark ML, Humphreys SM, et al. Effects of physiological hypercortisolemia on the regulation of lipolysis in subcutaneous adipose tissue. J Clin Endocrinol Metab. 1998;83: 626-31.

57. Gravholt CH, Schmitz O, Simonsen L, et al. Effects of a physiological GH pulse on interstitial glycerol in abdominal and femoral adipose tissue. Am J Physiol. 1999;277:E848-54.

58. Djurhuus CB, Gravholt $\mathrm{CH}$, Nielsen $\mathrm{S}$, et al. Effects of cortisol on lipolysis and regional interstitial glycerol levels in humans. Am J Physiol. 2002;283:E172-7.

59. Wee J, Charlton C, Simpson H, et al. GH secretion in acute exercise may result in post-exercise lipolysis. Growth Horm IGF Res. 2005;15:397-404.

60. Healy ML, Gibney J, Pentecost C, et al. Effects of high-dose growth hormone on glucose and glycerol metabolism at rest and during exercise in endurance-trained athletes. J Clin Endocrinol Metabol. 2006;91:320-7.

61. Tremblay M, Copeland J, Van Helder W. Influence of exercise duration on post-exercise steroid hormone responses in trained males. Eur J Appl Physiol. 2005;94:505-13.

62. Djurhuus CB, Gravholt $\mathrm{CH}$, Nielsen S, et al. Additive effects of cortisol and growth hormone on regional and systemic lipolysis in humans. Am J Physiol. 2004;286:E488-94.

63. Steensberg A, van Hall G, Osada T, et al. Production of interleukin-6 in contracting human skeletal muscles can account for the exercise-induced increase in plasma interleukin-6. J Physiol. 2000;529:237-42.

64. van Hall G, Steensberg A, Sacchetti M, et al. Interleukin-6 stimulates lipolysis and fat oxidation in humans. J Clin Endocrinol Metab. 2003;88:3005-10.

65. Hiscock N, Fischer CP, Sacchetti M, et al. Recombinant human interleukin-6 infusion during low-intensity exercise does not enhance whole body lipolysis or fat oxidation in humans. Am J Physiol. 2005;289:E2-7.

66. Wolsk E, Mygind H, Grøndahl TS, et al. IL-6 selectively stimulates fat metabolism in human skeletal muscle. Am J Physiol. 2010;299:E832-40.

67. Enevoldsen LH, Simonsen L, Macdonald IA, et al. The combined effects of exercise and food intake on adipose tissue and splanchnic metabolism. J Physiol. 2004;561:871-82.

68. Björkegren J, Karpe F, Milne RW, et al. Differences in apolipoprotein and lipid composition between human chylomicron remnants and very low density lipoproteins isolated from fasting and postprandial plasma. J Lipid Res. 1998;39:1412-20.

69. Grundy S, Mok H. Chylomicron clearance in normal and hyperlipidemic man. Metabolism. 1976;25:1225-39.

70. Xiang S-Q, Cianflone K, Kalant D, et al. Differential binding of triglyceride-rich lipoproteins to lipoprotein lipase. J Lipid Res. 1999;40:1655-62.

71. Karpe F, de Faire U, Mercuri M, et al. Magnitude of alimentary lipemia is related to intima-media thickness of the common carotid artery in middle-aged men. Atherosclerosis. 1998;141: 307-14.

72. Bickerton AST, Roberts R, Fielding BA, et al. Preferential uptake of dietary fatty acids in adipose tissue and muscle in the postprandial period. Diabetes. 2007;56:168-76.

73. Frayn K, Hodgetts V, Griffiths A. Mobilization and clearance of fat in exercising humans studied by regional venous catherization. In: Maughan R, Shirreffs S, editors. Biochemistry of exercise IX. Champaign: Human Kinetics; 1996. p. 73-88.

74. Sacchetti M, Saltin B, Olsen DB, et al. High triacylglycerol turnover rate in human skeletal muscle. J Physiol. 2004;561: 883-91.

75. Enevoldsen LH, Simonsen L, Bülow J. Postprandial triacylglycerol uptake in the legs is increased during exercise and postexercise recovery. J Physiol. 2005;568:941-50.

76. Harrison M, Moyna N, Zderic T, et al. Lipoprotein particle distribution and skeletal muscle lipoprotein lipase activity after acute exercise. Lipids Health Dis. 2012;11:64.

77. Seip R, Mair K, Cole T, Semenkovich C. Induction of human skeletal muscle lipoprotein lipase gene expression by short-term exercise is transient. Am J Physiol. 1997;272:E255-61.

78. Rattigan S, Wheatley C, Richards SM, et al. Exercise and insulinmediated capillary recruitment in muscle. Exerci Sport Sci Rev. 2005;33:43-8.

79. van Loon LJC, Greenhaff PL, Constantin-Teodosiu D, et al. The effects of increasing exercise intensity on muscle fuel utilisation in humans. J Physiol. 2001;536:295-304.

80. Weibel ER, Taylor CR, Hoppeler H. The concept of symmorphosis: a testable hypothesis of structure-function relationship. Proc Nat Acad Sci. 1991;88:10357-61.

81. Watt MJ, Holmes AG, Steinberg GR, et al. Reduced plasma FFA availability increases net triacylglycerol degradation, but not GPAT or HSL activity, in human skeletal muscle. Am J Physiol. 2004;287:E120-7.

82. Pernow B, Saltin B. Availability of substrates and capacity for prolonged heavy exercise in man. J Appl Physiol. 1971;31: 416-22.

83. Bonnard C, Durand A, Peyrol S, et al. Mitochondrial dysfunction results from oxidative stress in the skeletal muscle of diet-induced insulin-resistant mice. J Clin Invest. 2008;118:789-800.

84. Schrauwen P, Schrauwen-Hinderling V, Hoeks J, et al. Mitochondrial dysfunction and lipotoxicity. Biochim Biophys Acta. 2010;1801:266-71.

85. Nielsen JN, Wojtaszewski JFP, Haller RG, et al. Role of 5'AMPactivated protein kinase in glycogen synthase activity and glucose utilization: insights from patients with McArdle's disease. J Physiol. 2002;541:979-89.

86. Robertshaw HA, Raha S, Kaczor JJ, et al. Increased PFK activity and GLUT4 protein content in McArdle's disease. Muscle Nerve. 2008;37:431-7.

87. Vissing J, Duno M, Schwartz M, et al. Splice mutations preserve myophosphorylase activity that ameliorates the phenotype in McArdle disease. Brain. 2009;132:1545-52.

88. Haller RG, Vissing J. Spontaneous, "second wind" and glucoseinduced second "second wind" in McArdle disease: Oxidative mechanisms. Arch Neurol. 2002;59:1395-402.

89. Ørngreen M, Jeppesen T, Andersen S, et al. Fat metabolism during exercise in patients with McArdle disease. Neurology. 2009;72:718-24.

90. Andersen ST, Jeppesen TD, Taivassalo T, et al. Effect of changes in fat availability on exercise capacity in McArdle disease. Arch Neurol. 2009;66:762-6.

91. Kiens B, Essen-Gustavsson B, Christensen NJ, et al. Skeletal muscle substrate utilization during submaximal exercise in man: effect of endurance training. J Physiol. 1993;469:459-78. 